\title{
ON THE APPLICATION OF NEW CONVERGENCE CRITERIA FOR KANTOROVICH METHOD TO NONLINEAR SINGULAR INTEGRAL EQUATION WITH SHIFT
}

\author{
Mohamed M. Allan* and Smah M. Dardery \\ Department of Mathematics, Faculty of Education (Al Mahara), \\ Hadramut University of Science and Technology, Yemen. \\ *E-Mail: m_m_allan@hotmail.com
}

\begin{abstract}
The paper is concerned with the applicability of some new conditions for the convergence of Newton-kantorovich approximations to solution of nonlinear singular integral equation with shift of Uryson type. The results are illustrated in generalized Holder space.

Keywords: Newton-Kantorovich approximations, Nonlinear singular integral equations of Uryson type, Noether operator, Carleman shift.
\end{abstract}

\section{1- INTRODUCTION}

The theory of approximation methods and its applications for the solution of linear and nonlinear singular integral equations (LSIE) and (NSIE) has been developed by many authors $[5,11,12,17,21]$. There is a literature on the successful development of the nonlinear singular integral equations with shift (NSIES) $[1,3,4,15,18,20]$. The Noether theory of singular integral operators with shift (SIOS) is developed for a closed and open contour $([2,10,13,14,16,18]$ and others). The theory of singular integral equations with shift (SIES) is an important part of integral equations because of its recent applications in many fields of physics and engineering, [6,14,16]. It is known $[6,7]$, that Weiner-Hope equations are a natural apparatus for the solution of problems of synthesis of signais for linear systems with continuous time and stationary parameters. If the problem of synthesis is not stationary, then the Weiner-Hope method is not applicable and the problem is reduced to singular integral equation.

In this paper, some new conditions for the convergence of Newton-Kantorovich approximations have been applied to solution of the following NSIES of Uryson type:

$$
u(t)=\frac{1}{\pi i} \int_{\Gamma} \frac{k(\alpha(t), s, u(s))}{s-\alpha(t)} d s,
$$

in the generalized Holder space $H_{\Gamma}(\omega)$, where $\Gamma$ is a simple smooth closed Lyapunov contour, dividing the complex plane into two domains $D^{+}$( the interior domain) and $D^{-}$(the exterior domain), $D=D^{+} \cup D^{-}$, and the homeomorphism $\alpha: \Gamma \rightarrow \Gamma$ is the preserving orientation, satisfying the Carleman condition:

$$
\alpha(\alpha(t))=\alpha_{2}(t)=t ; \quad t \in \Gamma,
$$

and the derivative $\alpha^{\prime}(t) \neq 0$ satisfies the usual Holder condition. Moreover $k: \Gamma \times \Gamma \times \Re \rightarrow \Re$ is a caratheodory function (i.e $\mathrm{k}(., ., \mathrm{u})$ is measurable on $\Gamma \times \Gamma$ and $\mathrm{k}(\mathrm{t}, \mathrm{s},$.$) is continuous on \mathfrak{R})$. Also, we suppose that the derivative 


$$
l(t, s, u)=\frac{\partial k(t, s, u)}{\partial u}
$$

exists and is also a caratheodory function.

The special case of our problem has been studied as nonlinear integral equation with out shift in the Chebyshev space $\mathrm{C}$, the Lebesgue space $L_{p}(1 \leq p \leq \infty)$, and Orlicz space $L_{M}[8]$.

\section{2- FORMULATION OF THE PROBLEM:}

Let $X$ and $Y$ be two Banach spaces, $B\left(u_{0}, R\right)=\left\{u: u \in X,\left\|u-u_{0}\right\|<R\right\}$ the closed ball centered at $u_{0} \in X$ with radius $R>0$, and $F: B\left(u_{0}, R\right) \rightarrow Y$ is nonlinear operator. The Newton-Kantorovich method is one of the basic tools for finding approximate solutions of the operator

$$
F(u)=0
$$

In the corresponding iterative scheme

$$
u_{n+1}=u_{n}-F^{\prime}\left(u_{n}\right)^{-1} F\left(u_{n}\right), \quad(n=0,1,2, \ldots)
$$

One has torequire in particular that the Frechet derivative of $\mathrm{F}$ at all points $u_{n}$ exists and is invertible in the Banach space $C(X, Y)$ of all bonded linear operators from $X$ into $Y$. The non-negative numbers

$$
a=\left\|F^{\prime}\left(u_{0}\right)^{-1} F\left(u_{0}\right)\right\| \text {, and } b=\left\|F^{\prime}\left(u_{0}\right)^{-1}\right\|
$$

Will be of particular interest to us in what follows.

We suppose that the Frechet derivative $F^{\prime}(u)$ of $F$ satisfies at each point of $B\left(u_{0}, R\right)$ a condition of the form

$$
\left\|F^{\prime}\left(u_{1}\right)-F^{\prime}\left(u_{2}\right)\right\| \leq \mu\left(\left\|u_{1}-u_{2}\right\|\right), \quad u_{1}, u_{2} \in B\left(u_{0}, R\right)
$$

Where $\mu:[0, \infty) \rightarrow[0, \infty)$ is monotonically increasing with

$$
\lim _{r \rightarrow 0} \mu(r)=0, \quad 0<r<R
$$

Moreover, we assume that there is another monotonically increasing function $\theta:[0, \infty) \rightarrow[0, \infty)$ such that $0 \leq \theta(r) \leq \mu(r),(0 \leq r \leq R)$, and

$$
\left\|F^{\prime}(u)^{-1}\right\| \leq \frac{b}{1-b \theta(r)},\left(u \in B\left(u_{0}, r\right)\right) .
$$

We define three scalar functions on $[0, R]$ by

$$
\begin{aligned}
& \tilde{\mu}(r)=\sup \{\mu(u)+\theta(v): u+v=r\}, \\
& \phi(r)=\frac{a}{b}+\int_{0}^{r} \mu(t) d t-\frac{r}{b},(0 \leq r \leq R),
\end{aligned}
$$

and

$$
\widetilde{\phi}(r)=\frac{a}{b}+\int_{0}^{r} \tilde{\mu}(t) d t-\frac{r}{b},(0 \leq r \leq R) .
$$

As a special case of the main theorem of [12], about the convergence of successive approximations, we get then the following:

Theorem 2.1 [8]. Suppose that the function (2.8) has a unique zero $r_{0} \in[0, R]$ and that $\phi(R) \leq 0$. Then equation (2.1) has a solution $x_{*} \in B\left(x_{0}, r_{0}\right)$ this is unique in the ball $B\left(u_{0}, R\right)$ 
Lemma 2.1 [8]. Suppose that the function (2.9) has a unique zero $q_{*} \in[0, R]$ and that $\widetilde{\phi}(R) \leq 0$. Then the scalar sequence $\left(r_{n}\right)_{n \in N}$ defined by

$$
r_{0}=0, \quad r_{n+1}=r_{n}+\frac{b \widetilde{\phi}\left(r_{n}\right)}{1-b \theta\left(r_{n}\right)} \quad(n=0,1,2, \ldots)
$$

Converges monotonically to $q$ *

Theorem 2.2 [8]. Under the hypotheses of Lemma 2.1 the approximations (2.2) are defined for all $\mathrm{n}$ belong to the ball are converging to a solution of (2.1) and satisfy the estimates

$$
\left\|u_{n+1}-u_{n}\right\| \leq r_{n+1}-r_{n},(n=0,1,2, \ldots), \text { and }\left\|u_{*}-u_{n}\right\| \leq q_{*}-r_{n}, \quad(n=0,1,2, \ldots) .
$$

Theorem 2.3 [8]. Suppose that the sequence $\left(r_{n}\right)_{n}$ give by (2.10) converges to some limit $r_{\infty}(a)$. Then the approximations (2.2) are defined for all $\mathrm{n}$ belong to the ball $B\left(u_{0}, r_{\infty}(a)\right)$, and satisfy the estimate (2.11).

We remark that the usefulness of Theorem 2.2 consists in reducing the (hard) problem of finding zero of a nonlinear operator in a Banach space to the (possible simpler) problem of finding zero of a scalar function.

\section{3- SOME NOTATIONS AND AUXILIARY RESULTS:}

Definition 3.1 [10]. We denote by $\Phi$ the class of all functions $\omega(\delta)$, defined on $(0, l]$, where $l$ is the length of the curve ${ }_{\iota}$, which satisfies the following conditions:

1. $\omega(\delta)$ is a modulus of continuity,

$$
\text { 2. } \sup _{\delta>0} \frac{1}{\omega(\delta)} \int_{0}^{\delta} \frac{\omega(s)}{s} d s=I_{\omega}<\infty \text {, }
$$

3. $\sup _{\delta>0} \frac{\delta}{\omega(\delta)} \int_{\delta}^{l} \frac{\omega(s)}{s^{2}} d s=J_{\omega}<\infty$.

Definition $3.2[10,19]$. The generalized Holder space $H_{\Gamma}(\omega)$ is the set of all continuous function $u(t)$ such that

$$
H_{\Gamma}^{\omega}(u)=\sup \frac{\left|u\left(t_{1}\right)-u\left(t_{2}\right)\right|}{\omega\left(\left|t_{1}-t_{2}\right|\right)}<\infty .
$$

For $u(t) \in H_{\Gamma}(\omega)$, we define the norm

$$
\|u\|_{H_{\Gamma}}=\|u\|_{c(\Gamma)}+H_{\Gamma}^{\omega}(u) ; \quad\|u\|_{c(\Gamma)}=\max _{t \in \Gamma}|u(t)| .
$$

Definition $3.3[10,20]$. Let $\lambda_{1}$ and $\lambda_{2}$ be positive numbers and the function $\omega(t)$ satisfies the assumptions of Definition 3.1 we say that the function $u(t) \in H_{\Gamma}(\omega), t \in \Gamma$, belongs to the class $H_{\Gamma}^{\lambda_{1}, \lambda_{2}}(\omega)$ if the following two conditions are satisfied:

$$
\text { 1. }\|u(t)\|_{c} \leq \lambda_{1}, t \in \Gamma, \quad \text { 2. } H_{L}^{\omega}(u) \leq \lambda_{2} \text {. }
$$

Definition 3.4 $[16,19]$. Let $S: H_{\Gamma}^{\lambda_{1}, \lambda_{2}}(\omega) \rightarrow H_{\Gamma}^{\lambda_{1}, \lambda_{2}}(\omega)$, where $\lambda_{1}^{\prime}, \lambda_{2}^{\prime}$ are positive constants, denote to the operator of singular integration

$$
(S u)(t)=\frac{1}{\pi i} \int_{\Gamma} \frac{u(s)}{s-t} d s
$$

to which we associate the projection operators 


$$
P_{ \pm}=\frac{1}{2}(I \pm S), \quad S^{2}=I,
$$

where I is the identity operator on $H_{\mathrm{\Gamma}}(\omega)$. The Carleman shift operator

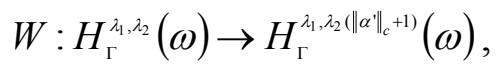

is given by

Using the notation

$$
(W u)(t)=u(\alpha(t))
$$

$$
(K u)(t)=\frac{1}{\pi i} \int_{\Gamma} \frac{k(\alpha(t), s, u(s))}{s-\alpha(t)} d s
$$

for nonlinear singular integral operator.

Lemma 3.1 [10]. Let the function $u(t)$ belong to the space $c(\Gamma)$ and $\int_{0}^{l} \frac{\omega_{u}(\xi)}{\xi} d \xi<\infty$.

Then the following inequalities

$$
\begin{gathered}
\|S u\|_{c} \leq c_{1}\left(\int_{0}^{\delta} \frac{\omega_{u}(\xi)}{\xi} d \xi+\|u\|_{c}\right) \\
\omega_{S u}(\delta) \leq c_{2}\left(\int_{0}^{\delta} \frac{\omega_{u}(\xi)}{\xi} d \xi+\delta \int_{0}^{\delta} \frac{\omega_{u}(\xi)}{\xi^{2}} d \xi\right)
\end{gathered}
$$

are valid, where $\omega_{u}(\delta)=\sup _{\substack{t_{1}-t_{2}<\delta \\ \delta>0}}\left|u\left(t_{1}\right)-u\left(t_{2}\right)\right|, c_{1}$ and $c_{2}$ are constants.

Lemma 3.2 [4]. The singular operator $S$ is a bounded operator on the space $H_{\Gamma}(\omega)$ and satisfies the inequality

$$
\|S u\|_{H_{\Gamma}} \leq \rho_{0}\|u\|_{H_{\Gamma}},
$$

where $\rho_{0}$ is a constant defined as follows $\rho_{0}=c_{1} \int_{0}^{\delta} \frac{\omega(\xi)}{\xi} d \xi+c_{1}+c_{2} \tilde{c} ; \tilde{c}$ is positive constant.

Lemma $3.3[4,20]$. The shift operator $W$ is a linear bounded continuously invertible operator on the space $H_{\Gamma}(\omega)$ and satisfies the inequality

$$
\|W u\|_{H_{\Gamma}} \leq \gamma_{0}\|u\|_{H_{\Gamma}}
$$

Where $\gamma_{0}=\max \left\{1, \alpha_{0}\right\}$ and $\alpha_{0}=\sup _{\delta>0} \frac{\omega_{u(\alpha(t))}(\delta)}{\omega_{u}(\delta)}$.

Now, we study the singular integral operator $K$ defined by the equality (3.4) where the function $k=k(t, s, u): \Gamma \times \Gamma \times R \rightarrow R$ satisfies the following condition

$$
\begin{aligned}
\left|k_{u^{(i)}}\left(t_{1}, s_{1}, u_{1}\right)-k_{u^{(i)}}\left(t_{2}, s_{2}, u_{2}\right)\right| \leq & A_{1}^{i} \omega^{*}\left(\left|t_{1}-t_{2}\right|\right)+A_{2}^{i} \omega\left(\left|s_{1}-s_{2}\right|\right), \\
& +\xi_{i}\left(\left|u_{1}-u_{2}\right|\right) ; i=0,1
\end{aligned},
$$

and for $\omega(\delta), \omega^{*}(\delta) \in \Phi$, we have

$$
\omega^{*}(\delta) \ln (l / \delta) \leq A_{3} \omega(\delta),
$$

$\xi_{i}:[0, \infty) \rightarrow[0, \infty)$ is monotonically increasing function with

$$
\lim _{r \rightarrow 0} \xi_{i}(r)=0 ; \quad 0 \leq r \leq R,
$$

where $A_{1}^{i}, A_{2}^{i}$ and $A_{3}$ are positive constants.

Lemma 3.4 [20]. If the inequalities (3.9)-(3.11) are satisfied, then for every $u(t) \in H_{\Gamma}^{\lambda_{1}, \lambda_{2}}(\omega)$, we have $(K u)(t) \in H_{\Gamma}^{\lambda_{\Gamma}^{k}, \lambda_{2}^{k}}(\omega)$, where $\lambda_{1}^{k}$ and $\lambda_{2}^{k}$ depend on $\lambda_{1}, \lambda_{2}$ 
Lemma 3.5. If the function $k(t, s, u)$ satisfies the conditions (3.9)-(3.11), then the operator $K$ defined by (3.4) is bounded on $H_{\Gamma}(\omega)$.

Proof

Let

$$
\begin{gathered}
k(t, s, u(s))=f_{1}(t, s, u(s))+k(s, s, u(s)) \\
f_{1}(t, s, u(s))=k(t, s, u(s))-k(s, s, u(s)) \\
\left|f_{1}\left(t_{1}, s_{1}, u\left(s_{1}\right)\right)-f_{1}\left(t_{2}, s_{2}, u\left(s_{2}\right)\right)\right| \leq A_{1}^{0} \omega^{*}\left(\left|t_{1}-t_{2}\right|\right)+\widetilde{A}_{2}^{0} \omega\left(\left|s_{1}-s_{2}\right|\right) \\
+\xi_{0}\left(\left|u\left(s_{1}\right)-u\left(s_{2}\right)\right|\right),
\end{gathered}
$$

Where $\widetilde{A}_{2}^{0}=2 A_{2}^{0}+A_{1}^{0} c_{1}(\ln (l / s))^{-1}$, hence, the norm of the operator $K$ can be rewritten as

$$
\begin{gathered}
\|K u\|_{H_{\Gamma}} \leq \gamma_{0}\|\widetilde{K} u\|_{H_{\Gamma}}=\gamma_{0}\left\{\|\widetilde{K} u\|_{c}+H_{\Gamma}^{\omega}(\widetilde{K} u)\right\}, \\
\|\widetilde{K} u(t)\|_{H_{\Gamma}} \leq\left\|\frac{1}{\pi i} \int_{\Gamma} \frac{f_{1}(t, s, u(s))}{s-t} d s\right\|_{H_{\Gamma}}+\rho_{0}\|k(t, t, u(t))\|_{H_{\Gamma}} .
\end{gathered}
$$

Let

$$
\begin{gathered}
M u(t)=\frac{1}{\pi i} \int_{\Gamma} \frac{f_{1}(t, s, u(s))}{s-t} d s, \\
\|M u\|_{c} \leq \frac{1}{\pi} \int_{\Gamma} \frac{\left|f_{1}(t, s, u(s))\right|}{|s-t|}|d s|,
\end{gathered}
$$

hence,

$$
\|M u\|_{c} \leq \frac{A_{1}^{0}}{\pi} \int_{\Gamma} \frac{\omega^{*}(|s-t|)}{|s-t|}|d s|
$$

It is well-known, [9], that for smooth contour $\Gamma$ there exist a number $m^{*}$ such that $|d s| \leq m^{*} d r$ where $m^{*}$ is a positive constant. From Definition 3.1, we have

$$
\|M u\|_{c} \leq \frac{m^{*} A_{3} A_{1}^{0}}{\pi \ln (l / \delta)} \int_{0}^{l} \frac{\omega(r)}{r} d r \leq \frac{m^{*} A_{3} A_{1}^{0}}{\pi \ln (l / \delta)} I_{\omega} \omega(l),
$$

Suppose $\left|t_{1}-t_{2}\right|<\sigma_{0}$, fix an arbitrary number $n, 1<n<\sigma_{0} /\left|t_{1}-t_{2}\right|$. Draw a circle of radius $\sigma=n\left|t_{1}-t_{2}\right|$ centered at the point $t_{1}$. This circle intersects $\Gamma$ at two points $\varepsilon_{1}$ and $\varepsilon_{2}$. The part of $\Gamma$ lying with this circle is denoted by $\varepsilon_{1} \varepsilon_{2}$.

From, [20], we obtain

$$
\begin{array}{ll} 
& \left|M u\left(t_{1}\right)-M u\left(t_{2}\right)\right| \leq \frac{1}{\pi}\left(2 q_{1}+q_{2}+q_{3}+q_{4}\right) \omega\left(\left|t_{1}-t_{2}\right|\right), \\
q_{1}=2 m *\left(\widetilde{A}_{2}^{0}+A_{4}\right)(n+1) I_{\omega}, & q_{3}=\left(\frac{n+1}{n}\right)^{2} m *\left(\widetilde{A}_{2}^{0}+A_{4}\right) J_{\omega}, \\
q_{2}=2 m * A_{1}^{0} A_{3}, & q_{4}=\left(A_{1}^{0} A_{3}(\ln (l / s))^{-1}+\widetilde{A}_{2}^{0}+A_{4}\right) M_{1},
\end{array}
$$

and $M_{1}=\left|\int_{\Gamma / \varepsilon_{\varepsilon_{2}}} \frac{d s}{s-t_{1}}-\pi_{i}\right|$, where, $A_{4}$ is positive constant, therefore, we have

$$
\begin{gathered}
\|M u(t)\|_{H_{\Gamma}} \leq \wedge_{1}+\wedge_{2}, \\
\wedge_{1}=\frac{m^{*} A_{3} A_{1}^{0}}{\pi \ln (l / s)} I_{\omega} \omega(l), \quad \wedge_{2}=\frac{1}{\pi}\left(2 q_{1}+q_{2}+q_{3}+q_{4}\right)
\end{gathered}
$$

Thene

$$
\|K u(t)\|_{H_{\Gamma}} \leq \gamma_{0}\left\{\wedge_{1}+\wedge_{2}+\rho_{0} \| K\left(t, t, u(t) \|_{H_{\Gamma}}\right\} .\right.
$$


Hence, the nonlinear singular integral operator $K$ defined by the right-hand side of (1.1) is a bounded operator in generalized Holder space $H_{\Gamma}(\omega)$. Therefore, we can rewrite equation (1.1) in the following form

$$
F(u)=u-K(u)=0, \quad\left(u \in \bar{B}\left(u_{0}, R\right)\right),
$$

for fixed $u \in H_{\Gamma}(\omega)$, we define an operator $L$ by

$$
L(u) h(t)=\frac{1}{\pi i} \int_{\Gamma}^{l(\alpha(t), s, u(s))} \frac{l}{s-\alpha(t)} h(s) d s ; \quad h(s) \in H_{\Gamma}(\omega),
$$

satisfied the following condition

$$
\left\|L\left(u_{1}\right)-L\left(u_{2}\right)\right\|_{H_{\Gamma}} \leq \mu\left(\left\|u_{1}-u_{2}\right\|_{H_{\Gamma}}\right), u_{1}, u_{2} \in B\left(u_{0}, R\right), 0<r<R,
$$

where

$$
\lim _{r \rightarrow 0} \mu(r)=0, \quad(0<r<R),
$$

and put

$$
G(u)=I-L(u), \quad\left(u \in \bar{B}\left(u_{0}, R\right)\right) .
$$

It is natural to expect that the derivative of the operator (3.4) is related to the operator (3.15), and hence the derivative of (3.14) is related to (3.17).

Lemma 3.6 [1]. Let the function $g(t, s)=m(t, s) h(s) ; h(s)$ belong to the generalized Holder space $H_{\Gamma}(\omega)$. Then the following inequality is valid

$$
\omega_{g}\left(\delta_{1}, \delta_{2}\right) \leq\|h\|_{c} \omega_{m}\left(\delta_{1}, 0\right)+\|h\|_{c} \omega_{m}\left(0, \delta_{2}\right)+\beta H_{\Gamma}^{\omega}(h) \omega(\delta),
$$

where

$$
\omega_{g}\left(\delta_{1}, \delta_{2}\right)=\sup _{\substack{t_{1}-t_{2}\left|<\delta_{1} \\ s_{1}-s_{2}\right|<\delta_{2}}}\left|g\left(t_{1}, s_{1}\right)-g\left(t_{2}, s_{2}\right)\right| \text {, and } \beta=\max _{t, s \in \Gamma}|m(t, s)| \text {. }
$$

The proof of boundedness of the operator $L$ in the generalized Holder space $H_{\Gamma}(\omega)$ depends on the inequality (3.6), [19].

In the following Theorem, the function $m=m(t, s)$ should carry the following quite restrictive conditions:

1. $\sup _{0<\delta<l} \frac{\omega_{m}(\delta, 0) \ln (l(\delta))}{\omega(\delta)}=I_{1}<\infty$

2. $\sup _{0<\delta<l} \frac{1}{\omega(\delta)} \int_{0}^{\delta} \frac{\omega_{m}(0, \xi)}{\xi} d \xi=I_{2}<\infty$

3. $\sup _{0<\delta<l} \frac{\delta}{\omega(\delta)} \int_{0}^{\delta} \frac{\omega_{m}(0, \xi)}{\xi^{2}} d \xi=I_{3}<\infty$

Theorem 3.1. The nonlinear singular operator $L$ is a bounded operator on the generalized Holder space $H_{\Gamma}(\omega)$.

Proof

$$
\text { Let } \quad m(t, s) h(s)=g(t, s) \text {, and } \widetilde{f}(t)=\frac{1}{\pi i} \int_{\Gamma} \frac{g(t, s)}{s-t} d s,
$$

where $l(t, s, u(s))=m(t, s)$, from, [1], we have

$$
\begin{array}{r}
\|\widetilde{f}\|_{c}=I_{4}\|h\|_{c}+I_{5} H_{\Gamma}^{\omega}(h), \text { and } H_{\Gamma}^{\omega}(\tilde{f}) \leq \widetilde{I}_{4}\|h\|_{c}+\widetilde{I}_{5} H_{\Gamma}^{\omega}(h), \\
I_{4}=m^{*} \int_{0}^{l} \frac{\omega_{m}(0, \xi)}{\xi} d \xi+\beta \pi, \quad \widetilde{I}_{4}=c_{2}\left(\frac{I_{1} I_{\omega}}{\ln 2}+I_{1}+I_{2}+I_{3}\right)
\end{array}
$$




$$
I_{5}=m * \beta \int_{0}^{l} \frac{\omega(s)}{\xi} d \xi, \quad \widetilde{I}_{4}=c_{2} \beta\left(I_{\omega}+J_{\omega}\right) .
$$

From, the inequalities (3.22) and (3.23), we obtain

$$
\begin{aligned}
& \|L(u) h\|_{H_{\Gamma}} \leq \theta\|h\|_{H_{\Gamma}} ; \\
& \theta=\gamma_{0} \cdot \max \left\{I_{4}+\widetilde{I}_{4}, I_{5}+\widetilde{I}_{5}\right\} .
\end{aligned}
$$

Thus, the theorem is proved.

Theorem 3.2. Suppose that the function

$$
K\left(u_{0}\right)(t)=\frac{1}{\pi i} \int_{\Gamma} \frac{k\left(\alpha(t), s, u_{0}(s)\right)}{s-\alpha(t)} d s,
$$

belongs to the space $H_{\Gamma}(\omega)$. Moreover suppose the operator $\mathrm{L}$ given by (3.15) is defined on the ball $\bar{B}\left(u_{0}, R\right)$, takes its values in the space of bounded linear operator on $H_{\Gamma}(\omega)$ and satisfies a condition (3.16a). Then the operator (3.14) is differentiable as an operator in $H_{\Gamma}(\omega)$ at every point $u \in B\left(u_{0}, R\right)$ and

\section{Proof}

$$
F^{\prime}(u)=G(u), \quad\left(u \in B\left(u_{0}, R\right)\right) .
$$

we remark that the condition (3.16a) implies that the operator $G$ is uniformly continuous on each ball $\bar{B}\left(u_{0}, r\right) \subset B\left(u_{0}, R\right)$. From the condition (3.25) and from

$$
k\left(\alpha(t), s, u(s)-k\left(\alpha(t), s, u_{0}(s)\right)=\int_{0}^{l} l\left(\alpha(t), s, u_{0}(s)+\lambda h(s)\right)(h(s)) d \lambda,\right.
$$

Where $h(s)=u(s)-u_{0}(s)$, hence

$$
K(u)(t)=K\left(u_{0}\right)(t)+L\left(u_{0}\right) h(t)
$$

It follows that also $K(u) \in H_{\Gamma}(\omega)$ for $\left\|u-u_{0}\right\|_{H_{\Gamma}} \leq R$; moreover,

$$
\|K(u)\|_{H_{\Gamma}} \leq\left\|K\left(u_{0}\right)\right\|_{H_{\Gamma}}+\left\|L\left(u_{0}\right)\right\|_{H_{\Gamma}}\left\|u-u_{0}\right\|_{H_{\Gamma}} .
$$

Frome definition of the function $k$ and its derivative and definition of the operator $L$, then the operator $F$ given by (3.14) is bounded as an operator from $\bar{B}\left(u_{0}, R\right)$ into $H_{\Gamma}(\omega)$. We show now that $F$ is differentiable with derivative G. Appling the definition of the operator $\mathrm{G}$ and changing the order of integration.

For fixed $u_{0}(t) \in H_{\Gamma}(\omega)$, and arbitrary element $h(t) \in H_{\Gamma}(\omega)$ we have the well-know formula:

$$
\begin{gathered}
F\left(u_{0}+h\right)-F\left(u_{0}\right)=G\left(u_{0}\right) h+\eta\left(u_{0}, h\right) \\
G\left(u_{0}\right) h(t)=h(t)-\frac{1}{\pi i} \int_{\Gamma}^{l\left(\alpha(t), s, u_{0}(s)\right)} \frac{l}{s-\alpha(t)} h(s) d s \\
\eta\left(u_{0}, h\right)=-\frac{1}{\pi i} \int_{\Gamma} \frac{1}{s-\alpha(t)}\left[\int_{0}^{1}\left\{l\left(\alpha(t), s, u_{0}(s)+\lambda h(s)\right)-l\left(\alpha(t), s, u_{0}(s)\right)\right\} h(s) d \lambda\right] d s \\
=\int_{0}^{1}\left[G\left(u_{0}+\lambda h\right)-G\left(u_{0}\right)\right] h(s) d \lambda .
\end{gathered}
$$

By hypothesis, the operator L satisfies the condition (3.16a), we have

$$
\left\|\eta\left(u_{0}, h\right)\right\|_{H_{\Gamma}} \leq \int_{0}^{1} \mu\left(\lambda\|h\|_{H_{\Gamma}}\right)\|h\|_{H_{\Gamma}} d \lambda=0\left(\|h\|_{H_{\Gamma}}\right),
$$

as $\|h\|_{H_{\Gamma}} \rightarrow 0$, and hence the equality (3.26) holds. 
Moreover, from the equalities (3.26), (3.29) and condition (3.16a), we get

$$
\left\|F^{\prime}\left(u_{1}\right)-F^{\prime}\left(u_{2}\right)\right\|_{H_{\Gamma}} \leq \mu\left(\left\|u_{1}-u_{2}\right\|_{H_{\Gamma}}\right),\left(u_{1}, u_{2} \in B\left(u_{0}, R\right)\right), o \leq r \leq R .
$$

\section{4- NOETHER PROPERTY AND INDEX FORMULA FOR SIOS:}

To study the Noether condition for the operator $G$, we reduce this operator to the following form

$$
G\left(u_{0}\right) h(t)=h(t)+\frac{d(t)}{\pi i} \int_{\Gamma} \frac{h(s)}{s-\alpha(t)} d s+\frac{1}{\pi i} \int_{\Gamma} R(t, \tau) h(s) d s=f(t)
$$

Where $d(t)=-l\left(\alpha(t), t, u_{0}(t)\right)$, and $R(t, s)=\frac{l\left(\alpha(t), t, u_{0}(t)\right)-l\left(\alpha(t), s, u_{0}(s)\right)}{s-\alpha(t)}$,

and $f \in H_{\Gamma}(\omega)$. From definition of the shift operator $W$ and singular integral operator $S$, we obtain

$$
G\left(u_{0}\right) h(t)=h(t)+d(t)(W S h)(t)+(N h)(t)=f(t),
$$

where $(N h)(t)=\frac{1}{\pi i} \int_{\Gamma} R(t, s) h(s) d s$. Using the relation (3.2), we get

$$
\begin{gathered}
G\left(u_{0}\right) h(t)=\left(A P_{+}+B P_{-}\right) h(t)=J(t) ; \\
A=I+d(t) W, B=I-d(t) W, \text { and } J(t)=f(t)-(N h)(t)
\end{gathered}
$$

From the theory of singular integral operators with shift [16], the Noether condition for the operator $\mathrm{G}$ is given by

$$
\Delta_{1}(t)=\left|\begin{array}{cc}
1 & d(t) \\
d(\alpha(t)) & 1
\end{array}\right| \neq 0, \quad \Delta_{2}(t)=\left|\begin{array}{cc}
1 & -d(t) \\
-d(\alpha(t)) & 1
\end{array}\right| \neq 0 .
$$

Moreover, the index formula of the operator $\mathrm{G}$ has the form

$$
\text { ind } G=\frac{1}{4 \pi}\left\{\arg \frac{\Delta_{2}(t)}{\Delta_{1}(t)}\right\}_{\Gamma} \text {. }
$$

\section{5- SOLUTION OF LINEAR SINGULAR INTEGRAL EQUATION WITH SHIFT:}

Now, we show that the linear singular integral equation with shift (4.2) has a unique solution for every $f \in H_{\Gamma}(\omega)$. Apply the operator $W S$ to both sides of the equation (4.2), hence, we obtain the following system:

$$
\begin{gathered}
h(t)+d(t)(W S h)(t)+(W h)(t)=f(t), \\
d(\alpha(t)) h(t)+(W S h)(t)+\left(N_{1} h\right)(t)=(W S f)(t)
\end{gathered}
$$

Where $N_{1}=W S d(t) W S-W d(t) W+W S N$

No solutions are lost when the operator $W S$ applied to equation (4.2), [1], hence all solutions of (4.2) are solutions of the system (5.1) and conversely. Let $E$ be the closed subspace defined by $E=\left\{(h, W S h), h \in H_{\Gamma}(\omega)\right\}$,

and let $\bar{\Omega}$ be the linear operator from $E$ to $H_{\Gamma}(\omega)$ defined by

$$
\begin{gathered}
\bar{\Omega} H(t)=\Omega(t) H(t), \\
H=\left[\begin{array}{c}
h \\
W S h
\end{array}\right], \quad \Omega(t)=\left[\begin{array}{cc}
1 & d(t) \\
d(\alpha(t)) & 1
\end{array}\right],
\end{gathered}
$$

where 
is a matrix of functions from the space $H_{\Gamma}(\omega)$ corresponding to the operator $\bar{\Omega}$. Moreover, if we put

$$
T=\left[\begin{array}{cc}
N & 0 \\
0 & N_{1} S W
\end{array}\right], \quad F=\left[\begin{array}{c}
f \\
W S f
\end{array}\right],
$$

then system (5.1) can be rewritten as the form:

$$
\bar{\Omega} H+T H=F, \quad H \in E
$$

Theorem 5.1 [1]. Assume that

$$
\operatorname{det} \Omega(t) \neq 0 \quad \forall t \in \Gamma, \quad\left\|\bar{\Omega}^{-1} T\right\|_{E}<1 .
$$

Then the operator $G\left(u_{0}\right)$ is invertible, moreover

$$
\left\|G\left(u_{0}\right)^{-1}\right\|_{H_{\Gamma}} \leq \frac{\left\|\Omega^{*}\right\|_{H_{\Gamma}}}{1-\left\|\Omega^{-1} T\right\|_{E}}\left(\frac{1}{n}+H_{\Gamma}^{\omega}(\lambda)\right),
$$

Where $n=\min _{t \in \Gamma}|\operatorname{det} \Omega(t)|, \lambda(t)=\frac{1}{\operatorname{det} \Omega(t)}$, and $\Omega^{*}$ be the adjoint matrix of $\Omega$. Assume

In fact, we have

$$
b=\frac{\left\|\Omega^{*}\right\|_{H_{\Gamma}}}{1-\left\|\Omega^{-1} T\right\|_{E}}\left(\frac{1}{n}+H_{\Gamma}^{\omega}(\lambda)\right), \quad a=b\left(\left\|u_{0}\right\|_{H_{\Gamma}}+\left\|K u_{0}\right\|_{H_{\Gamma}}\right)
$$

implies

$$
\begin{aligned}
L(u) & =L\left(u_{0}\right)+L(u)-L\left(u_{0}\right) \\
& =L\left(u_{0}\right)\left(I+L\left(u_{0}\right)\right)^{-1}\left(L(u)-L\left(u_{0}\right)\right),
\end{aligned}
$$

and consequently

$$
L(u)^{-1}=\left[I+L\left(u_{0}\right)^{-1}\left(L(u)-L\left(u_{0}\right)\right)\right]^{-1} L\left(u_{0}\right)^{-1},
$$

$$
\left\|L(u)^{-1}\right\|_{H_{\Gamma}} \leq \frac{\left\|L\left(u_{0}\right)^{-1}\right\|_{H_{\Gamma}}}{1-\left\|L\left(u_{0}\right)^{-1}\right\|_{H_{\Gamma}}\left\|L(u)-L\left(u_{0}\right)\right\|_{H_{\Gamma}}} .
$$

Put $\theta(r)=\sup \left\{\left\|L(u)-L\left(u_{0}\right)\right\|_{H_{\Gamma}}:\left\|u-u_{o}\right\|_{H_{\Gamma}} \leq r\right\}$,

where $\theta:[0, \infty) \rightarrow[0, \infty)$ such that $0 \leq \theta(r) \leq \mu(r),(0 \leq r \leq R)$. Similarly, we have

$$
\left\|G(u)^{-1}\right\|_{H_{\mathrm{\Gamma}}} \leq \frac{b}{1-b \theta(r)},\left(u \in B\left(u_{0}, r\right)\right) .
$$

Therefore, the following theorems are valid.

Theorem 5.2. Suppose that the function (2.8) has a unique zero $r_{*} \in[0, R]$ and that $\phi(R) \leq 0$. Then equation (3.14) has a solution $u_{*} \in B\left(u_{0}, r_{*}\right)$; this solution is unique in the ball $B\left(u_{0}, R\right)$.

Theorem 5.3. Under the hypotheses of Lemma 2.1 the approximation (2.2) are defined for all $\mathrm{n}$ belong to the ball $B\left(u_{0}, q_{*}\right)$, are converging to a solution $u_{*}$ of (3.14) and satisfy the estimate (2.11) .

\section{REFERENCES}

1. S. M. Amer and S. Dardery, On the theory of nonlinear singular integral equations with shift in Holder spaces, Forum Math 17, 753-780, 2005.

2. S. M. Amer and S. Dardery, On a class of nonlinear singular integral equations with shift on a closed contour, Appl. Math. And Comp. 158, 781-791, 2004. 
3. S. M. Amer and A. S. Nagdy, On the solution of a certain class of nonlinear singular integral and integro-differential equations with Carleman shift, Chaos, Solitons and Fractals 12, 1473-1484, 2001.

4. S. M. Amer, On solution of nonlinear singular integral equations with shift in generalized Holder space, Chaos, Solitons and Fractals 12, 1323-1334, 2001.

5. S. M. Amer, On the approximate solution of nonlinear singular integral equations with positive index, Int. J. Math. Math. Sci. 19 (2), 389-396, 1996.

6. A. A. Baturev, V. G. Kravchenko and G. S. Litvinchuk, Approximate method for singular integral equation with a non-Carleman shift; Integral Equation App 8, 1-17, 1996.

7. G. Cooper and C. Mcgillen, Probabilistic Methods of Singular and System Analysis, Holt, Rinehart and Winstons, Inc., New York, 1971.

8. E. De Pascale and P. P. Zabreiko, New convergence criteria for the NewtonKantorovich method and some applications to nonlinear integral equations, Rend. Sem. Mat. Univ. Padova 100, 1-20, 1998.

9. F. D. Gakhov, Boundary Value Problems; English Edition Pergamon Press Ltd, 1966.

10. A. I. Guseinov and Kh. Sh. Mukhtarov, Introduction to the Theory of Nonlinear Singular Integral Equations, Nauka Moscow (in Russian), 1980.

11. D. Jinyuan, The collocation methods and singular integral equations with Cauchy Kernel, Acta Math. Sci 20 (B3), 289-302, 2000.

12. L. V. Kantorovich and G. P. Akilov, Functiional Analysis; Pergamon Press. Oxford, 1982.

13. R. Sh. Khusnutdinov Solution of a class of nonlinear singular integro-differential equations with shift; Differential Equ. 25, 232-238, 1989.

14. V.G. Kravchenko, A.B. Lebre, G.S. Litvinchuk and F.S. Texeira, Fredholm theory for a class of singular integral operators with Carleman shift and unbounded coefficients, Math. Nach. 172, 199-210, 1995.

15. V. G. Kravchenko and G. S. Litvinchuk, Singular integral equations with Carleman linear fractional shift; Complex Variables, 26, 69-78,1994.

16. V.G. Kravchenko and G.S. Litvinchuk, Introduction to the Theory of Singular Integral Operators with Shift; Kluwer Academic Publishers, 1994.

17. E.G.Ladopoulous, V.A. Zisis, Nonlinear singular integral approximations in Banach spaces, Nonlinear Analysis, Theary, Methods Appl. 26(7), 1293-1299, 1996.

18. G. S. Litvinchuk, Boundary Value Problems and Singular Integral Equations with Shift; Nauka, Moscow, (in Russian), 1977.

19. S. G. Mikhlin and S. Prossdorf, Singular Integral Operator; Academy-Verleg, Berlin, 1986.

20. D. T. Nguyen, On a class of nonlinear singular integral equations with shift on complex curves, Acta Math. Vietnam 14 (2), 75- 92, 1989.

21. P. P. Zabrejko and D. F Nguen, The Majorant method in the theory of NewtonKantorovich approximations and the Ptak error estimates, Numer. Funct. Anal. And Optimize 9 (5 and 6), 671-684, 1987. 